\title{
Concurrent multiple brain tuberculomas with miliary tuberculosis - A rare presentation
}

\author{
Susmita Kundu', Aparup Dhua ${ }^{2}$, Saswata Ghosh ${ }^{3}$, Rajesh Agarwal ${ }^{4}$ \\ ${ }^{1}$ Professor and Head, Department of Pulmonary Medicine, R. G. Kar Medical College \& Hospital, Kolkata, India, ${ }^{2}$ Assistant Professor, \\ Department of Pulmonary Medicine, Midnapore Medical College \& Hospital, Paschim Midnapore, West Bengal, India, ${ }^{3}$ Assistant Professor, \\ Department of Pulmonary Medicine, Malda Medical College \& Hospital, Malda, West Bengal, India, ${ }^{4}$ Registrar, Critical Care Unit, Neotia \\ Getwel Hospital, Siliguri, West Bengal, India
}

\section{A B S T R A C T}

A 45 year old immunocompetent female presented to us with fever, dry cough, vomiting and headache. There was a single episode of convulsion after hospital admission. Chest Xray was normal. After through clinical examination and relevant investigations we came to know that it was a case of concurrent multiple brain tuberculomas with miliary tuberculosis. Patient was managed with antitubercular drugs and other supportive therapy promptly and she responed well. Occurence of tuberculoma in the brain in a patient with miliary tuberculosis is a rare phenomenon.
Access this article online

Website:

http://nepjol.info/index.php/AJMS

DOI: 10.3126/ajms.v6i2.10279

Key words: Tuberculoma, Miliary tuberculosis, Disseminated tuberculosis

\section{INTRODUCTION}

Although tuberculosis remains a major health problem in developing countries, tuberculoma involving the central nervous system is still uncommon compared with the involvement of other systems. ${ }^{1}$ Miliary tuberculosis, a form of disseminated tuberculosis occurs in 1-3 percent of all tuberculosis cases. ${ }^{2}$ Presence of multiple tuberculomas in brain associated with miliary tuberculosis is extremely rare, only few cases have been reported previously. ${ }^{3-6}$

\section{CASE REPORT}

A 45 year old female homemaker from an urban slum attended the out patient department with complaints of low grade fever, dry cough, persistent vomiting, headache, vertigo, fatigue along with loss of appetite for one month. Vital signs, such as pulse, blood pressure and temperature were normal. She was 1.52 meter tall and weighed $46 \mathrm{~kg}$ (Body mass index: 19.8). Pulmonary, neurological and other system examination were normal. After hospital admission there was a single episode of generalised tonic clonic seizure.
Laboratory investigations showed that haemoglobin was $11.6 \mathrm{gm} / \mathrm{dl}$, blood leukocyte was $8000 / \mathrm{mm}^{3}$ and erythrocyte sedimentation rate was $90 \mathrm{~mm}$ within first hour. Blood biochemical and urine examination were normal. Sputum was negative for acid fast bacilli by Zeihl-Neelsen staining after induction with nebulized hypertonic saline, for pyogenic organisms by Gram staining and for fungal elements by $\mathrm{KOH}$ mount. Mycobacterial culture by BACTEC method was negative. Chest Xray was within normal limits but high resolution computed tomography (HRCT) of the thorax (Figure 1) revealed innumerable small nodular lesions in both lung fields. Test for human immunodeficiency virus antibody was negative. Fiberoptic bronchoscopy was performed which showed no endobronchial lesion and bronchoalveolar lavage fluid was negative for acid fast bacilli both in smear and culture. Ultrasound of abdomen found no abnormality. Mantoux test reading was $15 \times 18 \mathrm{~mm}$. Bone marrow aspirate smear was also negative for acid fast bacilli. Multi Detector Computed tomography (MDCT) scan of brain revealed multiple small ill defined hypodense areas bilaterally without any remarkable mass effect. Contrast enhanced MRI scan of brain (Figure 2) detected multiple small ring enhancing lesions with surrounding perifocal vasogenic 


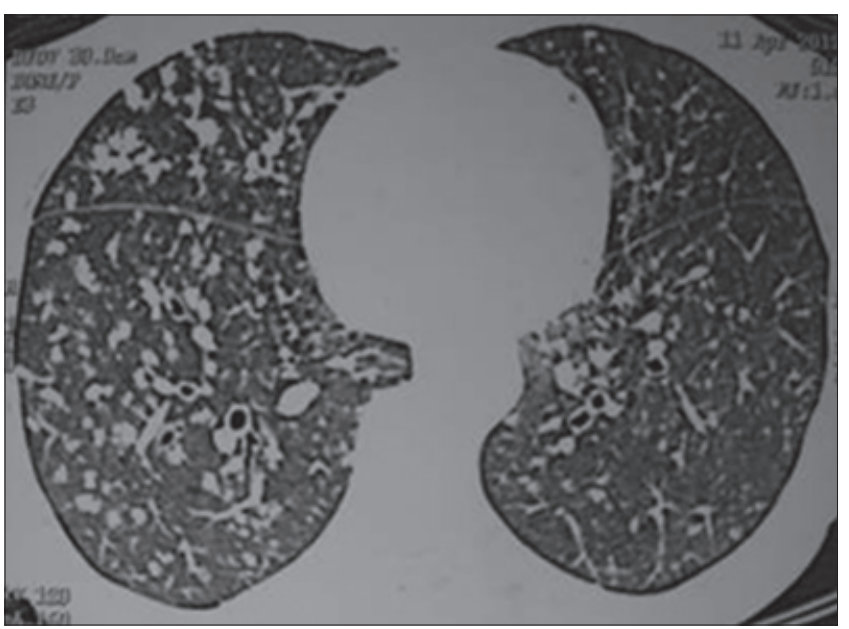

Figure 1: High resolution CT scan of thorax. HRCT thorax reveals innumerable small hyperdense nodular lesions in both lung fields

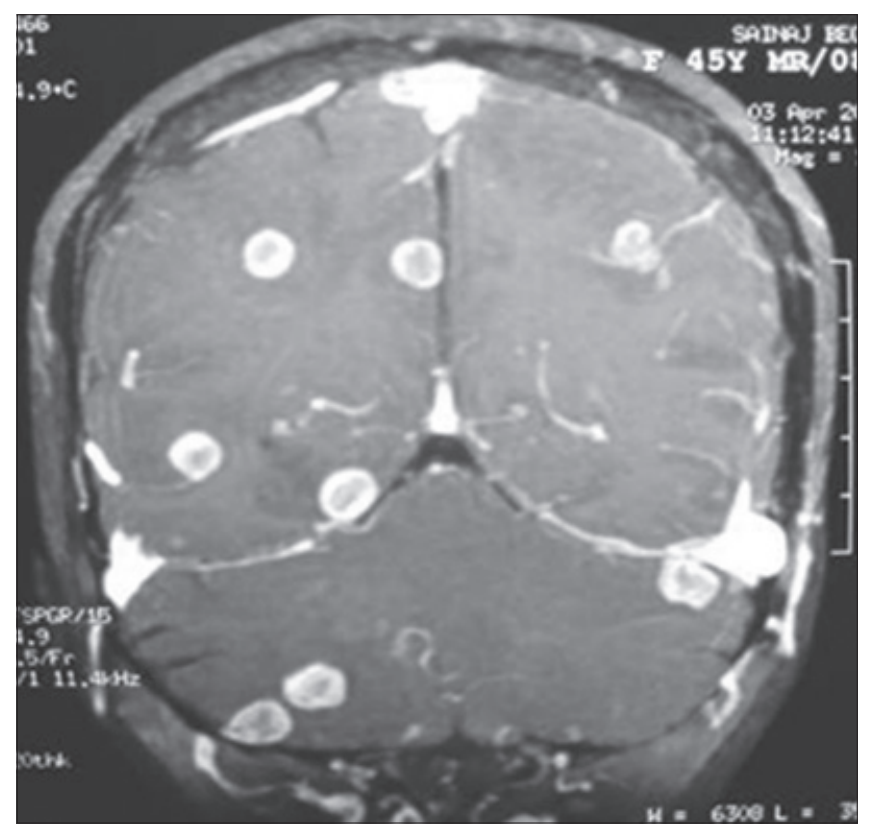

Figure 2: MRI (T1 weighted) of brain revealed multiple small ring enhancing lesion with surrounding oedema bilaterally

whitematter oedema bilaterally involving both hemisphere, cerebellum and pons. MR spectroscopy over the scattered lesions in both cerebral hemisphere was suggestive of non neoplastic lesions. Electro encephalogram revealed normal study. The fundus examination showed the presence of Choroid tubercles. Cerebrospinal fluid was lymphocytic with marginally raised adenosine deaminase activity. Antibodies to toxoplasma and VDRL test were negative.

The patient was diagnosed as miliary pulmonary tuberculosis with multiple brain tuberculomas and treatment started with a combination of four drugs (Isoniazid $600 \mathrm{mg}$, Rifampicin $450 \mathrm{mg}$, Pyrizinamide $1500 \mathrm{mg}$ and Ethambutol $1200 \mathrm{mg}$ ) thrice a week for initial two months and then two drugs (Isoniazid $600 \mathrm{mg}$ and Rifampicin $450 \mathrm{mg}$ ) thrice

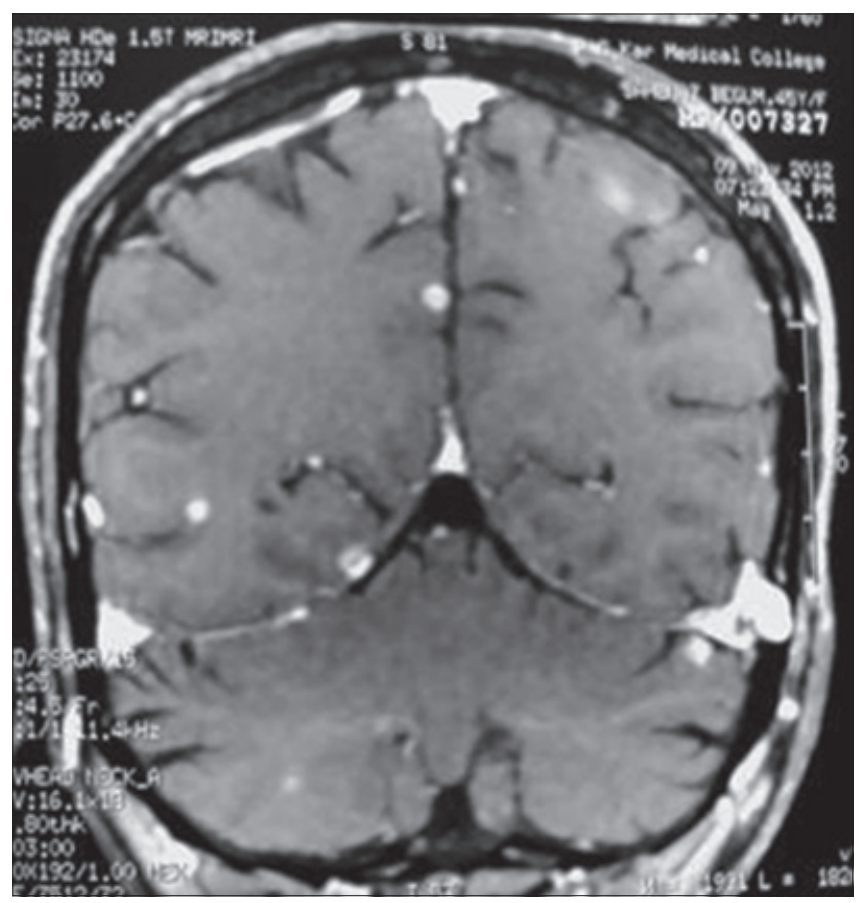

Figure 3: Post six months treatment MRI of brain revealed decrease in size of the lesions and totally reduced perilesional oedema

a week for next four months according to Category I treatment under Revised National Tuberculosis Control Programme in India. Initially Prednisolone $30 \mathrm{mg}$ once daily was started and then tapered over a period of eight weeks. Anti convulsant medication was continued. After six months of treatment patient had no symptoms, Chest Xray and HRCT thorax was normal and repeat Contrast enhanced MRI of brain revealed decrease in the size of the lesions and totally reduced perilesional oedema. Isoniazid and Rifampicin were extended for another six months according to provision of National programme.

\section{DISCUSSION AND CONCLUSION}

Tuberculosis shows very different clinical patterns depending on the organs it involves. Involvement of central nervous system occurs through hematogenous spread in the form of tubercular meningitis and tuberculoma. In developing countries, cerebro-meningeal involvement is a potentially lethal complication of tuberculosis. Evidence of active tuberculosis elsewhere in the body is seen in about $30-70 \%$ cases of brain tuberculomas. ${ }^{7}$ Only few reports describe miliary tuberculosis presenting with brain tuberculomas. ${ }^{3-6}$ Symptoms and signs of brain tuberculomas are generally only nonspecific like headache, dizziness, seizures, impairement of consciousness and focal neurological involvement. In this case convulsions occurred in the later part of disease without any neurodeficit. In our case brain tuberculomas probably occurred concurrently 
with the miliary tuberculosis as fever, dry cough and nonspecific central nervous system symptom were of same duration. Tuberculomas appear on brain CT scan as iso, hypo or hyperdense lesions, $1.5-7 \mathrm{~cm}$ diameter, with a peripheral enhancement after contrast enhancement and peripheral oedema. ${ }^{8}$ In MRI lesions appears isointense on pre-contrast T1 weighted images and with central hyperintense regions with hypointense rims on T2 weighted images. On contrast enhanced T1 weighted images the lesions appeared enhanced and some presented as ring enhanced lesions. ${ }^{9}$ Diagnosis of brain tuberculoma is difficult because the imaging presentation is varied and can be non-specific, other parameters may be required to establish the definite diagnosis. The most important factor in brain tuberculomas diagnosis is the suspicion of the disease. The CNS involvement in this case of miliary tuberculosis was suspected because of occurrence of seizure episode on the background of headache and dizziness. Diagnosis of brain tuberculoma was based on characteristic radiological lesions and good response to antituberculosis therapy. Miliary pattern on chest radiograph is the hallmark of miliary TB and is evident in a majority of patients. Before the advent of CT, it was observed that classical miliary pattern would not be evident in the chest radiograph in up to 50 per cent of the patients and would be detected only at the time of autopsy. ${ }^{10}$ HRCT thorax may reveal classical miliary or nodular pattern. Moreover intrathoracic lymphadenopathy, calcification and associated pleural or pericardial lesions may be detected during HRCT. Mantoux test positivity in certain number of cases of miliary $\mathrm{TB}$ like our case is also an interesting observation. ${ }^{11}$
Although rare multiple tuberculomas in brain can occur concurrently with miliary tuberculosis even with normal chest Xray, it requires high index of suspicion to diagnose and approach should be aggressive.

\section{REFERENCES}

1. Yen HL, Lee RJ, Lin JW and Chen HJ. Multiple tuberculomas in the brain and spinal cord: a case report. Spine 2003;28:E499-E502.

2. Sharma SK, Mohan A, Sharma A and Mitra DK. Miliary tuberculosis: new insights into an old disease.Lancet Infect Dis 2005; 5:415-430.

3. Ramchandran R, Kayanram $S$ and Prabhakar R. A rare presentation of miliary tuberculosis. Ind J Tub 1993;40:205.

4. Goel MK, Banavaliker JN, Singh P, Bhalotra B, Sharma SK and Ararwal RK. Miliary tuberculosis with brain tuberculomas a rare presentation. Ind J Tub 1997; 44:87-89.

5. Ceylan E and Gencer M. Miliary tuberculosis associated with multiple intracranial tuberculomas. Tohoku J Exp Med 2005;205;367-370.

6. Muin IA and Zurin AR. Pulmonary miliary tuberculosis with multiple intracerebral tuberculous granulomas--report of two cases. Br J Neurosur 1998;12:585-587.

7. Udani PM, Parekh UC and Dastur OK. Neurological and related symptoms in CNS tuberculosis. Neural Sc 1971;14:371.

8. Traub M, Colchester C F, Kinsley DPE and Swash M. Tuberculosis of central nervous system. Quart J Med 1984;209:80.

9. Gupta RK, Jena A, Sharma DK, Guha S, Khushu S and Gupta AK. MR imaging of Intracranial Tuberculomas. JCAT 1988;12:280-285.

10. Lee KS, Kim TS, Han J, Hwang JH, Yoon JH, Kim Y, et al. Diffuse micronodular lung disease: HRCT and pathologic findings. J Comput Assist Tomogr 1999; 23: 99-106.

11. Mert A, Bilir M, Tabak F, Ozaras R, Ozturk R, Senturk H, et al. Miliary tuberculosis: clinical manifestations, diagnosis and outcome in 38 adults. Respirology 2001;6:217-224. 